With 1 plate

Printed in Great Britain

\title{
Differentiation of Strains of Staphylococcus epidermidis Isolated From Bovine Udders
}

\author{
By R. W. BROWN, O. SANDVIK, R. K. SCHERER AND D. L. ROSE \\ National Animal Disease Laboratory, Animal Disease \\ and Parasite Research Division, Agricultural Research Service, \\ U.S. Department of Agriculture, Ames, Iowa, U.S.A.
}

(Accepted for publication 23 December 1966)

\begin{abstract}
SUMMARY
Strains of Staphylococcus epidermidis isolated from bovine udders were differentiated by comparing biochemical tests, serological typing of proteolytic enzymes, colonial morphology, and the spectrophotometric analysis of pigments extracted with methanol. The cultures were divided into proteinasepositive and proteinase-negative groups, based on their proteolytic activity on skim milk agar, staphylococcus medium no. 110 (Difco), and gelatin. Most of the proteinase-negative cultures produced acetoin, whereas the opposite was true for the proteinase-positive cultures. A further subdivision of the cultures in each group was made by using Baird-Parker's (1963) biochemical subgrouping scheme. The proteinase-positive cultures were also subdivided by serological typing of their proteolytic enzymes into five groups, B, F, G, H, and NR, a non-reacting group. Approximately three-quarters of the cultures in the first four proteinase groups could be placed in one of Baird-Parker's biochemical subgroups. The cultures in the NR group, however, were sufficiently distinctive in their biochemical reactions to be considered as a separate biochemical subgroup. The colonies formed by the cultures were classified into five types, each consisting of two to four forms. No absolute relationship was found between the spectral absorption curves of the pigments and proteinase groups, although there were differences in the types of absorption curves that predominated in each proteinase group. Nine cultures which produced coagulase were more closely related to $S$. epidermidis than to $S$. aureus in that they did not utilize mannitol anaerobically or produce $\alpha$ - or $\beta$-type haemolysis, they were non-pigmented and were less active biochemically. Also, serological typing of the proteolytic enzymes of three of these cultures resulted in one being classified as group $G$ and two as group $\mathbf{H}$. Group $\mathrm{H}$ contained only these two cultures. In contrast 27 cultures of $S$. aureus were classified as group A. Except for coagulase production, the biochemical reactions and the serological grouping of the proteolytic enzymes suggested a classification of $S$. epidermidis for the nine cultures.
\end{abstract}

\section{INTRODUCTION}

Staphylococcus epidermidis was found to be the principal cause of udder infections in a newly assembled research herd of Holstein-Friesian heifers. To study the pathogenicity and spread of the organisms in the herd, a differentiation of the strains was considered to be necessary. Differences in some characteristics of the organisms, such as colonial morphology, haemolysis, pigmentation, and biochemical reactions, indicated that the infections were caused by different strains; however, the tests used 
did not provide a good method of classification. Unlike $S$. aureus, $S$. epidermidis organisms are not typable with $S$. aureus bacteriophages, they do not produce specific toxins or haemolysins, and serological classification of cellular antigens has limited value since only two groups have been identified thus far (Losnegard \& Oeding, 1963). However, Baird-Parker $(1963,1965)$ has divided the staphylococci into six subgroups by physiological and biochemical tests, wherein subgroup I was similar to $S$. aureus and subgroups II to VI were similar to $S$. epidermidis. Sandvik \& Fossum (1965) have classified certain members of the Family Micrococcaceae by serological differentiation of their proteolytic enzymes. Pigmentation also has been studied as a means of differentiating strains of $S$. epidermidis by analysing the methanol-extracted pigments spectrophotometrically (Sandvik \& Brown, 1965) and by determining differences in the colour of their growth on Trypticase soy cream agar by spectral reflectance colrimetry (Brown, 1966).

This report, therefore, is concerned with the differentiation of strains of Staphylococcus epidermidis isolated from bovine udders by comparing physiological and biochemical tests, serological typing of proteolytic enzymes, colonial morphology, and the spectrophotometric analysis of methanol-extracted pigments. Strains of $S$. aureus isolated from bovine udders also were studied to provide a basis for comparison.

\section{METHODS}

Cultures. One hundred and ninety-two cultures of Staphylococcus epidermidis and 52 cultures of $S$. aureus were used in this study. All of the $S$. epidermidis and 42 of the $S$. aureus cultures were isolated from milk samples obtained from the experimental herd of cows sampled at weekly intervals over a 3-year period. The $S$. epidermidis cultures were isolated from 81 quarters of 34 cows and the $S$. aureus cultures from 18 quarters of 12 cows. Seven of the cows were infected in 10 quarters with both species at different times. Each milk sample was streaked fresh $(0 \cdot 1 \mathrm{ml}$.) on a bovine blood agar plate which was incubated for $24 \mathrm{hr}$ at $37^{\circ}$ and for $24 \mathrm{hr}$ at room temperature (about $23^{\circ}$ ). Each culture was selected from a single representative colony and transferred to two tubes of beef infusion broth. After incubätion at $37^{\circ}$ for $24 \mathrm{hr}$, one tube was stored at $-65^{\circ}$ and the other used to inoculate media for preliminary identification.

Cultures were classified as Staphylococcus aureus when they were Gram-positive cocci which produced catalase and coagulase, utilized glucose and mannitol anaerobically and produced $\alpha$-, $\alpha \beta$ - or $\beta$-type haemolysis. Cultures were classified as $S$. epidermidis when they were Gram-positive cocci which produced catalase but not coagulase, utilized glucose but not mannitol anaerobically, and produced slight or no haemolysis.

When the detailed study was begun, each frozen culture was thawed and $0 \cdot 1 \mathrm{ml}$. transferred to a tube of beef infusion broth and incubated for $20-24 \mathrm{hr}$ at $37^{\circ}$. Each culture was then streaked on $5 \%(\mathrm{v} / \mathrm{v})$ bovine blood agar and incubated for $24 \mathrm{hr}$ at $37^{\circ}$ and for $24 \mathrm{hr}$ at room temperature. The colonies were graded as positive or negative for pigment, and the type of morphology noted for those cultures classified as Staphylococcus epidermidis. A representative colony was transferred to a Tryptose agar (Difco Laboratories, Detroit, Michigan, U.S.A.) slope which was incubated at $37^{\circ}$ for $24 \mathrm{hr}$ and then stored at $4^{\circ}$ and used for subcultures. 
Unless otherwise specified, all test media were inoculated from a fresh $24 \mathrm{hr}$ Tryptose Agar slope culture and tests were read directly or performed on $1 \mathrm{ml}$. samples of culture after 2, 5 and 7 days of incubation at $37^{\circ}$. Enzyme activity on skim milk agar, tributyrin agar, egg yolk agar and staphylococcus medium no. 110 (Difco) was determined by the development of either a zone of clearing or a zone of precipitation around the point of inoculation. In most tests, the reactions are reported as negative or positive for ease in making comparisons, even though various gradations of reactions were observed and recorded.

Blood agar. Washed red blood cells from $10 \mathrm{ml}$. of bovine blood were added to $200 \mathrm{ml}$. melted beef infusion agar (beef infusion; peptone, $1 \% ; \mathrm{NaCl}, 0.5 \%$; agar, $2 \%$ ) which had been cooled at $45^{\circ}$.

Acid production from carbohydrates. Anaerobic acid production was determined by making stab cultures in phenol red glucose and mannitol agars (Difco) which contained $1 \%$ carbohydrate. Before use, the media were steamed at $100^{\circ}$ for $10 \mathrm{~min}$. to drive off oxygen and then cooled at $4^{\circ}$. The cultures were incubated anaerobically in Brewer jars in an atmosphere of hydrogen for 5 days.

Aerobic acid production was determined in phenol red glucose, mannitol, lactose and maltose broths (Difco) each containing $1 \%$ carbohydrate.

Coagulase production. One volume of citrated rabbit plasma was diluted with 4 vol. of a sodium chloride solution $(0.85 \%, \mathrm{w} / \mathrm{v})$ and $0.5 \mathrm{ml}$. of the mixture was used for each test. After inoculation, the tubes were incubated for $4 \mathrm{hr}$ at $37^{\circ}$ and a further $20 \mathrm{hr}$ at room temperature. Readings were made after incubation for 1, 4 and $24 \mathrm{hr}$.

Phosphatase production. A $1 \%$ solution of phenolphthalein diphosphate (trisodium salt, Sigma Chemical Co., St Louis, Missouri, U.S.A.) was sterilized by filtration through a Seitz filter (ST-3) and added to sterile beef infusion broth to give a final concentration of $1 / 10,000$. To test for phosphatase activity, one drop of $\mathrm{N}$-sodium hydroxide solution was added to a $1 \mathrm{ml}$. sample of the broth culture. A crimson red colour was recorded as positive and a pink colour as weak positive.

Urease production. Cultures were grown on urea agar (Difco) slopes and the decomposition of urea graded as positive when the entire medium turned red and weak positive when only a part of the medium turned pink.

Acetoin production. Cultures were grown in MR-VP medium (Difco) and acetoin was detected in $1 \mathrm{ml}$. samples by adding $1 \mathrm{ml}$. of modified O'Meara's reagent $(0.3 \%$ solution of creatine in $40 \%$ potassium hydroxide; Manual of Microbiological Methods, 1957). All cultures which were negative after incubation for 7 days were also tested by adding $0.5 \mathrm{ml}$. of $5 \% \alpha$-naphthol in $95 \%(\mathrm{v} / \mathrm{v})$ ethanol in water, and $0.5 \mathrm{ml}$. of $0.1 \%$ creatine in $16 \%$ potassium hydroxide to $1 \mathrm{ml}$. of culture (Page, 1962). In both tests, the mixtures were incubated at room temperature and visual readings made after $4 \mathrm{hr}$.

Nitrate reduction. Cultures were grown in nitrate broth (Difco) and $1 \mathrm{ml}$. of culture tested for nitrites by adding a drop of sulphanilic acid and a drop of $\alpha$-naphthylamine reagent (Manual of Microbiological Methods, 1957). All negative tests were confirmed by adding zinc dust to determine whether nitrate was still present or had been decomposed beyond the nitrite stage.

Asparagine utilization. Cultures were grown on slopes of a medium containing asparagine, $0 \cdot 1 \%$; glucose, $1 \%$; potassium chloride, $0.02 \%$; magnesium chloride, $0.02 \%$; agar, $1.5 \%$ and bromcresol purple, $0.0016 \%$ (Bergey's Manual, 1957, footnote, 
p. 455). Slopes were examined only for acid production and graded as positive when the entire medium was yellow and weak positive when the surface of the slope was partially or completely yellow.

Gelatin liquefaction. Nutrient gelatin (Difco) in screw-capped tubes was inoculated and incubated at $30^{\circ}$ for 4 weeks. Examination for liquefaction was made at weekly intervals after the tubes had been refrigerated at $4^{\circ}$ for $30 \mathrm{~min}$. Cultures were considered negative for gelatinase activity when there was no liquefaction or only a thin layer of liquid on top, weakly positive when only the top $\frac{1}{2}$ in. $(0 \cdot 7-1 \cdot 3 \mathrm{~cm}$.) of medium was liquid, and positive for all other degrees of liquefaction.

Skim milk agar. The medium was prepared by adding $25 \mathrm{ml}$. of fresh skim milk to $100 \mathrm{ml}$. of hot beef infusion agar, mixing, and then pouring into Petri plates. The milk was obtained aseptically from a non-infected udder, centrifuged, and then skimmed. Sixteen cultures were point-inoculated on each plate. The plates were incubated at $30^{\circ}$ for $48 \mathrm{hr}$ and proteinase activity graded according to the size of the zone of precipitation (Sandvik, 1962).

Staphylococcus medium no. 110 ( $S$-110 medium). Four cultures were tested on each Petri plate, containing $12 \mathrm{ml}$. medium, by making a streak about $1 \frac{1}{2}$ in. $(4 \mathrm{~cm}$.) long from the periphery toward the centre of the plate with a loopful of a $24 \mathrm{hr}$ broth culture. After the plates had been incubated at $30^{\circ}$ for $48 \mathrm{hr}$, the amount of growth was graded as good (growth confluent), fair (growth not confluent and with a nodular appearance), poor (growth sparse or only to be seen with a hand lens), or negative. Cultures which showed good growth were also graded as smooth or rough according to the type of growth. Smooth growth appeared moist and glossy, and rough growth usually appeared dry with the edges frequently irregular. Final classification of the type of growth was based on its wettability. When the surface of the medium was flooded with a $20 \%$ sulphosalicylic acid solution to test for gelatinase activity, the liquid readily flowed over the smooth growth on immediate contact; but the rough growth remained unwetted until about $10 \mathrm{ml}$. of liquid had been added to each plate. The rough growth frequently separated from the medium and floated in the liquid.

Gelatinase production was determined, 10-15 min. after the addition of the sulphosalicylic acid, by grading the amount of clearing around each culture.

Tributyrinase production. A mixture of $2 \mathrm{ml}$. of a $1 \%$ solution of polysorbate 80 (Tween 80, Atlas Chemicals, Wilmington, Delaware, U.S.A.), $1 \mathrm{ml}$. of tributyrin (Eastman Organic Chemicals, Rochester, New York, U.S.A.), and $16 \mathrm{ml}$. of an $0.85 \%$ solution of sodium chloride was autoclaved at $121^{\circ}$ for $15 \mathrm{~min}$. After being autoclaved, the hot mixture was shaken vigorously for $1 \mathrm{~min}$. to emulsify the tributyrin. The emulsion was then mixed with $80 \mathrm{ml}$. of melted beef infusion agar and poured into Petri plates. Sixteen cultures were point-inoculated on each plate. The plates were incubated for $48 \mathrm{hr}$ and tributyrinase activity was indicated by a zone of clearing around the culture. This is the medium described by Marks (1952) except that beef infusion agar was substituted for nutrient agar.

Egg yolk agar. A $10 \%$ suspension (v/v) of fresh egg yolk was made aseptically in beef infusion broth. The suspension was heated to $50^{\circ}$ and mixed with an equal volume of beef infusion medium containing 3\% agar. The melted beef infusion agar was cooled to $50^{\circ}$ before adding the egg yolk and pouring the mixture into Petri plates. Sixteen cultures were point-inoculated on each plate. The plates were incubated for 
$48 \mathrm{hr}$ and 'lipase' activity was indicated by the formation of a cloudy zone usually surrounded by a clear or lytic zone. A pearly surface sometimes developed within the cloudy zone.

Colonial morphology. All studies on colonial morphology were made with broth cultures that were spread on blood agar plates and incubated at $37^{\circ}$ for $24 \mathrm{hr}$ and at room temperature for a further $24 \mathrm{hr}$. The classification of colonial types was based on single colonies that were sufficiently separated from other growth to permit maximum development of size and shape. Photographs of representative colonies were used for comparison and the colonial morphology of each culture was designated by the identification number of the culture(s) they resembled.

Pigments. Pigments were extracted from whole organisms with hot methanol and examined spectrophotometrically (Sandvik \& Brown, 1965). The spectral absorption curves were classified into seven types, designated I, II, III, IV, V, VI, and a Staphylococcus aureus type. Two subtypes were included in each of types I and III; all non-pigmented extracts were classified as type I. The extracts from 95 cultures of $S$. epidermidis were classified by this method.

Serological classification of proteinases. The cultures were classified for proteinase activity on the basis of their reactions on skim milk agar, nutrient gelatin and S-110 medium. Cultures that were positive on any one of the three media were subdivided into groups according to the serological specificity of the proteinase as determined by the inhibition of enzyme activity by a specific antiserum (Sandvik \& Fossum, 1965). The specific antiproteinase was separated from normal proteinase inhibitors in immune rabbit sera by paper electrophoresis. The paper strip was then applied to a caseinate medium; after incubation the strip was removed from the medium and replaced by narrower strips of filter paper that had been immersed in solutions containing the proteinases to be tested. The antiproteolytic effect of the antiserum was demonstrated when the specific enzyme was prevented from precipitating the sodium caseinate in the area of the $\gamma$-globulin. Liquefied gelatin was used as a source of the enzyme for each culture. When cultures did not liquefy gelatin, enzymes were obtained by growing the organisms on a skim milk agar (agar concentration $0.8-0.9 \%$; Sandvik, 1962). When this study was begun there were available five specific antisera, designated A, B, C, D, E (Sandvik \& Fossum, 1965). However, three additional antisera were prepared during the study and designated F, G, H. Cultures were classified proteinase-negative when they showed no reaction with all three of the media used to determine proteinase activity.

\section{RESULTS}

\section{Biochemical studies}

The biochemical reactions of Staphylococcus aureus and S. epidermidis cultures grown on various media are compared in Table 1 . Eight cultures which were classified as $S$. aureus met all of the requirements that were used for classifying them in this species except that, they did not ferment mannitol (two cultures), or they produced only slight haemolysis (four cultures), or they showed a combination of both characteristics (two cultures). We considered them to be $S$. aureus because they were variants of strains which fermented mannitol and produced $\alpha \beta$-type haemolysis. Nine cultures which produced coagulase were not included with the $S$. aureus cultures in Table 1 
because they were more like $S$. epidermidis in growth characteristics and metabolic activity. Consequently, these cultures are treated as a coagulase-positive group in Tables 2-4 with the $S$. epidermidis cultures.

The Staphylococcus aureus cultures gave more positive reactions than the $S$. epidermidis cultures in all the tests made. The greatest difference between the two species occurred with those tests used routinely for their differentiation (i.e. coagulase, haemolysis and fermentation of mannitol).

Table 1. A comparison of biochemical reactions of cultures of Staphylococcus aureus and $S$. epidermidis

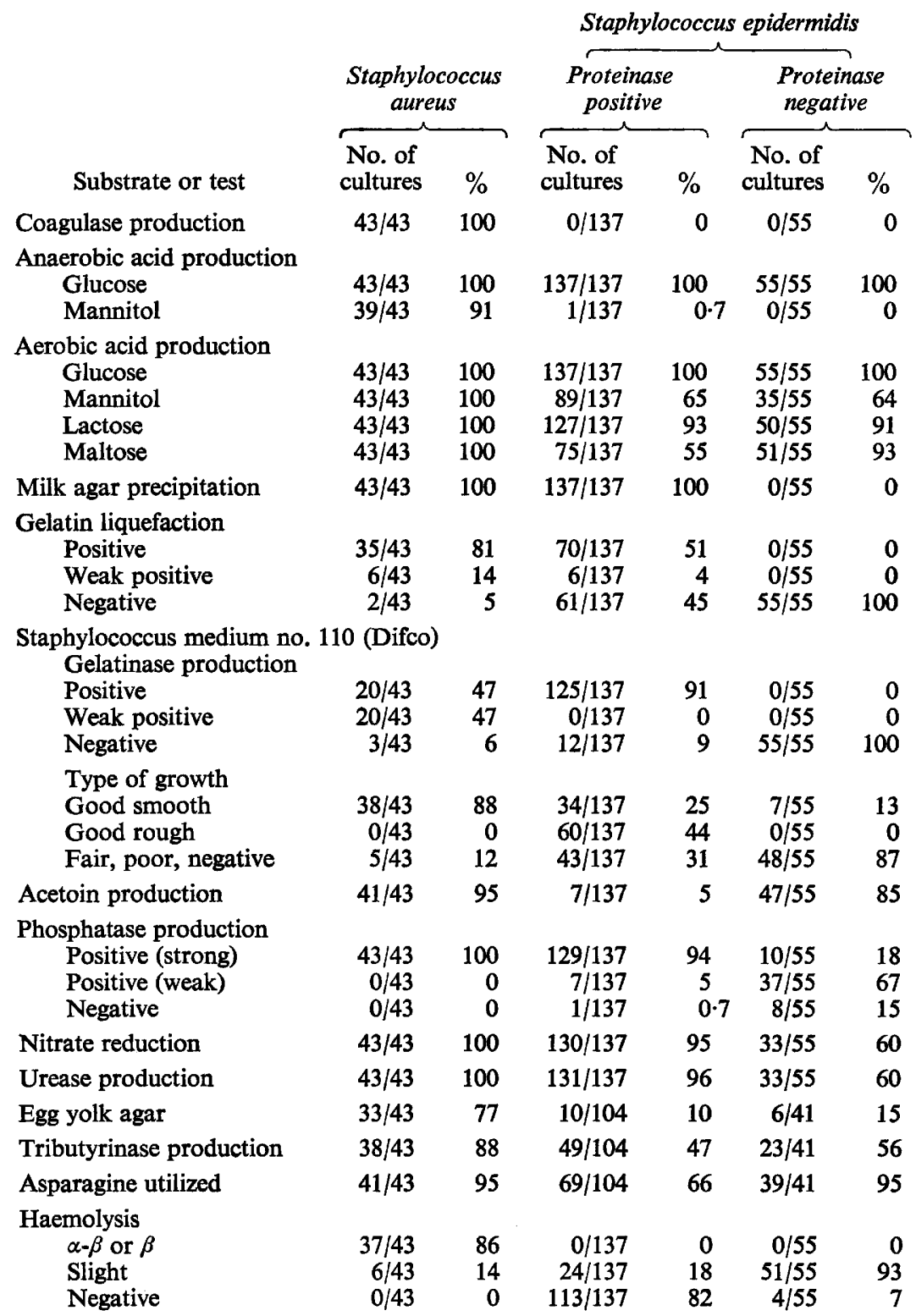


The Staphylococcus epidermidis cultures were divided into proteinase-positive and proteinase-negative groups to demonstrate metabolic differences within the species based on this classification (Table 1). Both groups showed comparable results for the aerobic utilization of glucose, mannitol and lactose, and for lipolytic activity on egg yolk agar and tributyrin agar. The proteinase-positive cultures, considered as a group, were able to grow better on S-110 medium and gave approximately one-third (30-40\%) more positive reactions than did the proteinase-negative cultures in the reduction of nitrate to nitrite and in the production of urease. However, the proteinase-positive cultures were less active than the proteinase-negative cultures in utilizing maltose aerobically, and in utilizing asparagine as the sole source of nitrogen. The principal differences between the two groups were found for the production of phosphatase, acetoin, and haemolysis.

Phosphatase. All except one of the proteinase-positive cultures were positive for phosphatase. A strong positive test was given by $87 \%$ of these cultures after 2 days of incubation and by an additional $7 \%$ of cultures after 7 days. The other $5 \%$ produced only weak positive reactions. Although $85 \%$ of the proteinase-negative cultures were positive for phosphatase, a strong positive reaction for phosphatase was produced by only $18 \%$ of the cultures; $13 \%$ after 2 days and the other $5 \%$ after 5-7 days. Those cultures which made up the $13 \%$ were also the only proteinasenegative cultures that developed good smooth growth on S-110 medium. In all instances, when a culture gave a weak positive test at the end of 2 days it always gave a strong positive test by the fifth day. Also, all cultures that were classified as weakly positive for phosphatase after 7 days never produced the weak positive reaction until after 5 or 7 days.

For comparative purposes, 34 proteinase-negative cultures which had been tested previously for phosphatase activity in liquid medium, were tested on an agar medium described by Baird-Parker (1963). The cultures that gave negative (5), weak positive (20), and late strong positive reactions (3) in the liquid medium were all negative on the agar medium. However, the 6 cultures that were strongly positive for phosphatase in the liquid medium after 2 days of incubation were also positive on the agar medium.

Acetoin. The proteinase-negative cultures were more active in the production of acetoin. Only $5 \%$ of the proteinase-positive cultures gave a positive test for acetoin with modified O'Meara's reagent, as opposed to $85 \%$ of the proteinase-negative cultures. When a more sensitive test was used (Page, 1962), the number of positive reactions increased to 17 and $94 \%$ for the proteinase-positive and proteinase-negative cultures, respectively. However, five-sixths of these additional positive reactions were graded as trace or weak. With the more sensitive test, the strong positive reactions developed as soon as the reagents were added to the culture medium, whereas the trace and weak positive reactions developed later. Comparable results with the two tests can be obtained when the less sensitive test is read after $4 \mathrm{hr}$ and the more sensitive test after $30 \mathrm{~min}$.

Haemolysis. The Staphylococcus epidermidis cultures were either non-haemolytic or slightly haemolytic. Only $18 \%$ of the proteinase-positive cultures were slightly haemolytic as opposed to $93 \%$ of the proteinase-negative cultures. The slight haemolysis appeared primarily as either a small zone $(<1 \mathrm{~mm}$.) of complete or partial clearing around the colony, or as a relatively large area of partial haemolysis that was 
lighter than the surrounding medium. The latter haemolysis gave the appearance of a halo and was distinguished from $\beta$-type haemolysis wherein the zone of affected red blood cells is well demarcated and appears darkened. A larger well demarcated zone of clearing that extended 1-2 mm. beyond the edge of the colony also was produced by $3 \%$ of the $S$. epidermidis cultures.

\section{Proteinase grouping}

Serology. One hundred and ten of the 137 proteinase-positive cultures of Staphylococcus epidermidis and 28 of the cultures of $S$. aureus were classified by serological differentiation of their proteolytic enzymes with specific antiserums. All of the $S$. aureus cultures were classified as group A except one, which did not fall into any group. This culture was coagulase positive, produced $\alpha \beta$ haemolysis but did not utilize mannitol anaerobically and was a weak producer of proteinase. Sixty-six of the $S$. epidermidis cultures were placed in one of three groups; 50 in B, eight in F, and eight in G. The other 44 cultures produced proteolytic enzymes that did not react with any of the antisera and were classified in a non-reacting (NR) group.

A comparison of the cultures of Staphylococcus epidermidis separated into the various serological groups showed that the cultures in each group were related in metabolic activity (Table 2). Included in Table 2 are seven of eight tests used by Baird-Parker (1963) as a basis for his biochemical grouping of staphylococci (i.e. coagulase, phosphatase and acetoin production, fermentation of mannitol, and oxidation of mannitol, lactose and maltose) plus gelatin liquefaction and growth on S-110 medium, which we found useful in classifying the cultures. The nine cultures that produced coagulase were placed in a separate group to show their close relationship to $S$. epidermidis. Two of these cultures were typed as group $\mathrm{H}$ and one as group $\mathrm{G}$ while the other six were not tested serologically. Three cultures in group NR were omitted because their biochemical and growth characteristics were sufficiently different to indicate they were not related to the other 41 cultures.

Proteinase-positive cultures. In Table 2, the cultures in groups B and NR showed the greatest differences in their biochemical reactions and growth characteristics. The group B cultures were the least active and the NR cultures the most active in the aerobic utilization of mannitol and maltose. Although $36 \%$ of the B cultures were graded as positive for the aerobic utilization of mannitol, two-thirds of these reactions were weakly positive. Almost all of the B cultures liquefied gelatin $(98 \%)$ but none of the NR cultures, even though all of the latter group were gelatinase-positive on S-110 medium. Only the B cultures produced fair, poor, or no growth on S-110 medium, whereas all of the NR cultures produced good rough growth. Although approximately half of the B cultures were graded as producing good smooth growth, the surface of the growth was irregular rather than homogeneous, and differed from the nodular appearance of fair growth only in that it was more extensive and coalescent. The cultures in group F were intermediate to those in groups B and NR and were the only proteinase positive cultures that were positive for acetoin with O'Meara's reagent. A close relationship between the cultures in groups $G$ and NR was indicated by the fact that five of the eight group G-cultures gave test reactions identical to those given by the cultures in group NR. The coagulase positive cultures in Table 2 differed from the Staphylococcus aureus cultures in Table 1 by not utilizing mannitol anaerobically or mannitol and maltose aerobically, by not producing acetoin, and by not producing 
$\alpha$ or $\beta$ haemolysis. All of these cultures reduced nitrate, produced urease, and liquefied gelatin, except the two group $\mathrm{H}$ cultures.

Proteinase-negative cultures. These cultures were divided into two subgroups on the basis of the type of growth on S-110 medium and the production of acetoin and phosphatase (Table 2). The cultures in subgroup 1 gave fair, poor, or no growth on S-110 medium, positive tests for acetoin, and late strong positive, weak positive, or negative tests for phosphatase. The cultures in subgroup 2 gave good, smooth growth on S-110 medium, negative tests for acetoin, and strong tests for phosphatase after 2 days of incubation.

Table 2. A comparison of biochemical reactions of various groups of proteinase positive and proteinase negative cultures of Staphylococcus epidermidis

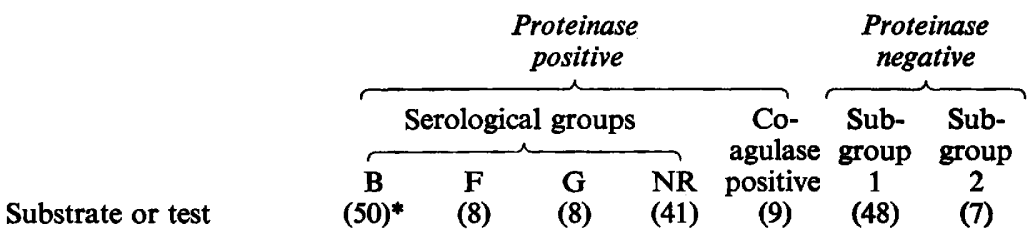

(8) (41)

(9)

(48)

\section{Coagulase production \\ Phosphatase production \\ Positive (strong) \\ Positive (weak) \\ Negative}

Acetoin production

Acid production

Mannitol (anaerobic)

Mannitol (aerobic)

Lactose (aerobic)

Maltose (aerobic)

Gelatin liquefaction
Percentage of cultures positive

\begin{tabular}{rrrrrrr}
\hline 0 & 0 & 0 & 0 & 100 & 0 & 0 \\
100 & 100 & 100 & 98 & 100 & 6 & 100 \\
0 & 0 & 0 & 2 & 0 & 77 & 0 \\
0 & 0 & 0 & 0 & 0 & 17 & 0 \\
0 & 75 & 0 & 0 & 0 & 98 & 0 \\
& & & & & & \\
0 & 0 & 0 & 0 & 0 & 0 & 0 \\
36 & 38 & 100 & 100 & 0 & 65 & 57 \\
100 & 75 & 62 & 98 & 100 & 90 & 100 \\
4 & 100 & 100 & 100 & 0 & 98 & 57 \\
98 & 76 & 0 & 0 & 78 & 0 & 0
\end{tabular}

Staphylococcus medium 110 (Difco) Type of growth

Good smooth

Good rough

Fair, poor, negative,

$\begin{array}{rrrrrrr}48 & 63 & 12 & 0 & 100 & 0 & 100 \\ 0 & 37 & 88 & 100 & 0 & 0 & 0 \\ 52 & 0 & 0 & 0 & 0 & 100 & 0\end{array}$

* Number of cultures per group.

\section{Colonial morphology}

A study of the colonial morphology of Staphylococcus epidermidis was made to determine the different colonial forms that developed, to classify into types the forms which showed similarities in their structure and development, and to determine any relationship between the colonial types and proteinase groups. Preliminary studies showed that the colonies formed on blood agar by cultures isolated directly from raw milk were smaller and in some instances rougher, drier, and more irregular in shape than the colonies formed after the cultures were grown in beef infusion broth and re-isolated on blood agar. The effect on the colonial morphology was attributed to the raw milk and not to a change that developed after the cultures had been isolated and grown in broth. This was shown when smaller irregular colonies were produced by suspending the growth from broth cultures in raw milk and then spreading $0 \cdot 1 \mathrm{ml}$. 
of this milk on a blood agar plate. The effect of raw milk was nullified by diluting the milk 10 times with $0.85 \%$ sodium chloride solution before inoculating the blood agar.

The colonies formed by the cultures of Staphylococcus epidermidis were classified into five types, each consisting of two to four forms (Plate 1). More than one form of a type might develop on the same agar plate. The surface of most colonies appeared moist (glossy finish) although some appeared dry (mat finish).

The S-1 type consisted of colonies which were smooth and with entire margins. The differentiation into four forms was based on surface variations that consisted of the presence or absence of a raised centre and ring formations caused by differences in coloration between the centre and peripheral zones. When growth of the other $S$ types was heavy, the colonies frequently appeared as S-1 type.

Type S-2 colonies were smooth with entire margins and characterized by a peripheral zone that was well demarcated from the central area of the colony. The two forms were differentiated by the degree of demarcation, with form $(b)$ showing the most demarcation and a distinct dome-shaped central area. The peripheral zone was lighter in colour in both pigmented and non-pigmented colonies and in some instances was translucent. Form $(b)$ with a translucent peripheral zone was associated more frequently with small colonies. A slightly depressed area developed in the centre of some colonies which gave the appearance of a small dark circle.

Type S-3 colonies were smooth with finely serrate or undulate margins and the two forms were differentiated by the degree of irregularity of the margin. Some colonies showed raised centres while others appeared to have a flat central area surrounded by a peripheral collar.

Table 3. A comparison of the types of colonies associated with the various groups of Staphylococcus epidermidis

\begin{tabular}{|c|c|c|c|c|c|c|}
\hline \multirow{2}{*}{$\begin{array}{l}\text { Colonial } \\
\text { morphology } \\
\text { type }\end{array}$} & \multicolumn{4}{|c|}{ Serological groups } & \multirow[b]{2}{*}{$\begin{array}{l}\text { Coagulase } \\
\text { positive } \\
\text { (9) }\end{array}$} & \multirow[b]{2}{*}{$\begin{array}{l}\text { Proteinase } \\
\text { negative } \\
\text { (55) }\end{array}$} \\
\hline & $\stackrel{\mathrm{B}}{(50)^{*}}$ & $\begin{array}{c}F \\
(8)\end{array}$ & $\begin{array}{c}G \\
(8)\end{array}$ & $\begin{array}{l}\mathrm{NR} \\
(41)\end{array}$ & & \\
\hline $\begin{array}{l}\text { S-1 } \\
\text { S-2 } \\
\text { S-3 } \\
\text { S-1 } \\
\text { R }\end{array}$ & $\begin{array}{r}1 \\
49 \\
0 \\
0 \\
0\end{array}$ & $\begin{array}{l}2 \\
0 \\
0 \\
3 \\
3\end{array}$ & $\begin{array}{l}1 \\
0 \\
2 \\
0 \\
5\end{array}$ & $\begin{array}{r}3 \\
0 \\
35 \\
0 \\
3\end{array}$ & $\begin{array}{l}9 \\
0 \\
0 \\
0 \\
0\end{array}$ & $\begin{array}{r}44 \\
0 \\
0 \\
11 \\
0\end{array}$ \\
\hline
\end{tabular}

Type s-1 colonies were smooth with entire margins and were classified separately from the S-1 type because of their small size. They had the appearance of a flat smooth button and were differentiated into two forms on the basis of the presence or absence of ring formations caused by differences in coloration between the centre and peripheral zones.

Type $\mathrm{R}$ colonies were rough with irregular lobate margins. The four forms were differentiated by the degree of lobation and the degree of wrinkling of the surface in the central area of the colony.

A comparison of the colonial types produced by the organisms in each group is presented in Table 3. A relationship in the type of colony produced by the 
cultures in each group existed for the cultures in the serological groups B and NR, the coagulase-positive and the proteinase-negative cultures. All of the cultures in group B, except one, produced S-2 type colonies, the majority $(85 \%)$ of the cultures in group NR produced S-3 type colonies, the nine coagulase-positive cultures produced S-1 type colonies, and the proteinase-negative cultures produced either S-1 or s-1 type colonies. All of the proteinase-negative cultures in subgroup 2 produced S-1 type colonies. No relationship existed between the colonial type and proteinase group for the cultures in groups $F$ and $G$, although the cultures in group $G$ produced the same three colony types as the cultures in group NR.

\section{Table 4. A comparison of the types of spectral absorption curves associated with the various groups of Staphylococcus epidermidis}

\begin{tabular}{|c|c|c|c|c|c|c|}
\hline \multirow[b]{2}{*}{$\begin{array}{l}\text { Type of spectral } \\
\text { absorption curve* }\end{array}$} & \multicolumn{4}{|c|}{ Serological groups } & \multirow{2}{*}{$\begin{array}{c}\text { Coagulase } \\
\text { positive } \\
\text { (9) }\end{array}$} & \multirow{2}{*}{$\begin{array}{c}\text { Proteinase } \\
\text { negative } \\
\text { (31) }\end{array}$} \\
\hline & $\underset{(13) \dagger}{\mathbf{B}}$ & $\begin{array}{c}F \\
(8)\end{array}$ & $\begin{array}{l}G \\
(8)\end{array}$ & $\begin{array}{l}\text { NR } \\
(26)\end{array}$ & & \\
\hline I (non-pigmented) & 0 & 8 & 4 & 2 & 9 & 4 \\
\hline II & 4 & 0 & 2 & 0 & 0 & 10 \\
\hline III & 0 & 0 & 1 & 20 & 0 & 3 \\
\hline IV & 2 & 0 & 1 & 4 & 0 & 0 \\
\hline V & 1 & 0 & 0 & 0 & 0 & 12 \\
\hline VI & 5 & 0 & 0 & 0 & 0 & 0 \\
\hline S. aureus & 1 & 0 & 0 & 0 & 0 & 2 \\
\hline
\end{tabular}

\section{Pigments}

The spectral absorption curves of the pigments extracted with methanol were determined for 95 cultures of Staphylococcus epidermidis. A comparison of the absorption curves produced by the cultures in each proteinase group is given in Table 4. No absolute relationship existed between these two characteristics, although there were differences in the types of absorption curves that predominated in each proteinase group. The predominant types of absorption curves associated with groups $\mathrm{B}, \mathrm{NR}$, and proteinase-negative cultures were II and VI, III, and II and V, respectively. In addition, seven of the eight cultures in group $\mathrm{F}$ and the nine coagulase-positive cultures were non-pigmented. The one culture in group $\mathrm{F}$ was so poor in pigment production that colonies developed only a trace of pigment on tryptose agar and the spectral absorption curve produced with methanol-extracted pigment was classified as type Ib. Only the group B cultures produced the type VI curve, and all of the type $\mathrm{V}$ curves, except one, were produced by proteinase-negative cultures. The type II and VI curves were associated with poor pigment production since the extracts had to be concentrated threefold before satisfactory curves could be obtained.

\section{DISCUSSION}

All of the cultures of Staphylococcus epidermidis and most of the cultures of $S$. aureus used in this study were isolated from the udders of cows in one dairy herd. The results, therefore, may be biased, in that all cultures with similar characteristics, 
regardless of their source, could be considered to be the same strain. Consequently, the results with any of the tests performed could have been influenced by how well any one strain spread in the herd and by its frequency of isolation and inclusion in the group of cultures tested. In spite of this, we feel that the cultures used in this study are representative of the strains of $S$. epidermidis that infect bovine udders. However, any conclusions made about the differentiation of this species apply only to bovine strains.

In most instances, the separation of the cultures into Staphylococcus aureus and $S$. epidermidis was accomplished on the basis of coagulase production and the anaerobic utilization of glucose and mannitol. However, nine cultures that produced coagulase were more closely related to $S$. epidermidis than to $S$. aureus, for the following reasons. These nine cultures did not utilize mannitol anaerobically; they did not produce $\alpha$ - or $\beta$-type haemolysis and were non-pigmented; they gave biochemical reactions, except for coagulase, similar to Baird-Parker's subgroup III rather than subgroup I, which contained the $S$. aureus (Baird-Parker, 1963); the proteolytic enzymes of three of the nine cultures tested were serologically classified as groups $\mathrm{G}$ and $\mathrm{H}$, whereas the $S$. aureus cultures in this study and those studied by Sandvik \& Fossum (1965) were classified in group A. In addition, the two group H cultures were resistant to lysis with $S$. aureus bacteriophages when tested by Dr E. H. Coles (College of Veterinary Medicine, Kansas State University, Manhattan, Kansas, U.S.A.). The possibility that the nine cultures did not produce coagulase but coagulated citrated rabbit plasma by utilizing citrate was disproved by the fact that they also coagulated heparinized rabbit plasma. These organisms are not strains of $S$. aureus which have lost certain characteristics while being maintained in the laboratory. For instance, two of the cultures were representative of 47 and 50 isolates obtained separately at weekly intervals from two infected quarters. All of these isolates were tested for coagulase, haemolysis, pigment, and anaerobic utilization of glucose and mannitol: all showed the same characteristics as the nine cultures described above.

According to Baird-Parker (1965), these cultures would be classified as Staphylococcus aureus since they are positive in two of the three tests he recommended for recognition of this species (i.e. coagulase, phosphatase, fermentation of mannitol). He suggested that further confirmation of diagnosis can be obtained by determining phage typability, haemolysin pattern and ability to grow in normal human serum. The latter test was not used with our cultures, but the results in the other two tests would not add any support to the classification of $S$. aureus. Regardless of their classification, these nine organisms are sufficiently different for them to be easily distinguished from the usual strains of $S$. aureus that infect bovine udders. However, if $\alpha \beta$ - or $\beta$-type haemolysis is the only criterion used for the identification of staphylococci, as is frequently used in the routine diagnosis of udder infections, these cultures would be diagnosed as non-haemolytic or non-pathogenic staphylococci or just micrococci.

As a result of this work, we feel that the determination of proteolytic activity is valuable in the differentiation of strains of Staphylococcus epidermidis. This is best done on skim milk agar; however, testing for the liquefaction of gelatin and gelatinase production on S-110 medium is useful in confirming the proteolytic activity of a culture, if a weak reaction is produced on the milk agar. The liquefaction of gelatin is the poorest indicator of proteolytic activity. The 41 cultures in the NR group were unique, as a group, in that they were unable to liquefy gelatin in the tube yet they 
produced gelatinase on S-110 medium. It was thought that this resulted because, either the optimum temperature of incubation was not used, or conditions in the tube were insufficiently aerobic. When six cultures of this group were grown in nutrient gelatin medium in tubes and on Petri plates and incubated at $20^{\circ}, 30^{\circ}$ and $37^{\circ}$, none of the cultures liquefied the gelatin. Apparently this medium is not conducive to the production of proteolytic enzymes by this group of organisms.

Use of the S-110 medium was also of value in showing differences in growth characteristics of the organisms. The difference between the cultures that gave good smooth growth and good rough growth may be caused by a greater amount of lipid in the organisms of the rough-growing cultures. This opinion is based on the observation that the rough type of growth was unwetted by a $20 \%$ sulphosalicylic acid solution or by water when either was added to the surface of the solid medium and allowed to flow over the plate, whereas the same type of growth was readily wetted and covered by light petroleum.

Acetoin production was found to be useful as a differential test by Abd-el-Malek \& Gibson (1948) and was used by Shaw, Stitt \& Cowan (1951) to subdivide the coagulasenegative staphylococci. In some respects, a division of our Staphylococcus epidermidis cultures based on acetoin production would give a breakdown similar to our proteinase positive and proteinase-negative groups (with some exceptions), in that most of the proteinase-positive cultures were acetoin negative and most of the proteinase-negative cultures were acetoin positive (Tables 1,2 ). Of course, more exceptions occur when the test for acetoin is a more sensitive one. Our experience with two tests of different sensitivity tends to confirm the observation made by Shaw et al. (1951) that a test can be too sensitive and lose its differential value. Although we did only a limited study, a similar situation developed when two phosphatase tests were compared. The method which uses a broth medium was more sensitive than a method which uses an agar medium (Baird-Parker, 1963). Nevertheless, the latter method may be better because the weak phosphatase-producing cultures appear negative on the agar medium, which permits a simple positive or negative classification of cultures.

By utilizing seven of the eight tests proposed by Baird-Parker (1963) for grouping staphylococci, a comparison was made of the reactions given by the cultures in our groups with the reaction scheme given for his (B-P) subgroups. The test not made by us and omitted from the comparisons was the determination of growth at $10^{\circ}$.

All of the cultures in the proteinase group A were classified in the B-P subgroup I which contained the Staphylococcus aureus. Only four cultures in group F were classified as B-P subgroup II and none of the cultures was classified as B-P subgroup IV. Thirty group $B$ cultures $(60 \%)$ and three cultures of the proteinase-negative subgroup 2 could be placed in B-P subgroup III. The coagulase-positive cultures, not classified as $S$. aureus, showed agreement with B-P subgroup III in all tests except coagulase. Almost all ( $91 \%$ ) of the proteinase-negative cultures of our subgroup I could be placed in B-P subgroups V (33\%) and VI $(58 \%)$ when the weak positive phosphatase reactions were considered comparable to a negative rather than to a positive reaction. This seemed justified in view of the fact that 21 of these cultures gave negative tests for phosphatase when tested by Baird-Parker's method. None of the group G or NR cultures could be placed in any Baird-Parker subgroup.

It would seem that by dividing bovine strains of Staphylococcus epidermidis into proteinase-positive and proteinase-negative groups, further subdivision can then be 
made by using Baird-Parker's biochemical subgrouping scheme or by serological grouping of the proteolytic enzymes (Sandvik \& Fossum, 1965). When Baird-Parker's 8-test scheme is used for classification, consideration should be given to using staphylococcus medium no. 110 (Difco) and the liquefaction of gelatin as supplementary tests. Because the cultures in the proteinase NR group were so distinctive, and because they did not fit any of Baird-Parker's staphylococcal subgroups, it might be desirable to consider them as an additional subgroup, based on the reactions given in. Table 2 . It should be realized that these biochemical reactions do not represent all of the proteinase-positive cultures that fall into the NR group. Consequently, proteinasepositive NR cultures should be further differentiated into biochemical groups. If we exclude the group NR cultures from consideration, approximately three-quarters of the $S$. epidermidis cultures that were typed could be placed in one of Baird-Parker's biochemical subgroups. However, the difficulty of relying on the biochemical method alone for the differentiation of strains of $S$. epidermidis is indicated by the fact that some of the proteinase-negative subgroup 2 cultures were classified as B-P subgroup III along with proteinase group B cultures. In addition, $36 \%$ of the proteinase group B cultures were excluded from subgroup III because they utilized mannitol aerobically.

The colonial types described by us for Staphylococcus epidermidis are not unlike the descriptions given by Shaw et al. (1951) for the staphylococci. They stated that colonies were round, low, convex and opaque and that most strains produced colonies with a smooth surface and an entire edge; but a minority had rough-surfaced colonies and a few produced colonies with undulate or crenated edges. It would seem that we differ only in further differentiating their most common form into S-1, S-2 and s-1 types. Their rough-surfaced colonies and colonies with undulate edges would correspond to our types R and S-3, respectively. Although colonial morphology may have little value in the classification of staphylococci, it can be useful in differentiating strains of $S$. epidermidis.

The value of determining the spectrophotometric absorption curves of pigments and the colour of pigmented growth as methods of differentiating strains of Staphylococcus epidermidis has been presented in other reports (Sandvik \& Brown, 1965; Brown, 1966). The data presented in Table 4 further emphasize the problem of using one method for differentiating strains of $S$. epidermidis. The pigment studies, however, have proved valuable in showing the presence of the same type of organism in the same infected quarter of an udder during a long period.

The authors express appreciation to Mr R. M. Glazier, Illustration and Photographic Services, National Animal Disease Laboratory, for preparing the illustrations in this paper. This investigation was supported by a U.S. Public Health Service International Postdoctoral Fellowship (G-V-III).

\section{REFERENCES}

Abd-El-MaleK, Y. \& Gibson, T. (1948). Studies in the bacteriology of milk. II. The staphylococci of milk. J. Dairy Res. 15, 249.

BAIRD-PARKer, A. C. (1963). A classification of micrococci and staphylococci based on physiological and biochemical tests. J. gen. Microbiol. 30, 409.

Baird-Parker, A. C. (1965). The classification of staphylococci and micrococci from world-wide sources. J. gen. Microbiol. 38, 363.

Bergey's Manual of Determinative Bacteriology (1957). 7th ed. p. 445, footnote. Baltimore: Williams \& Wilkins Co. 

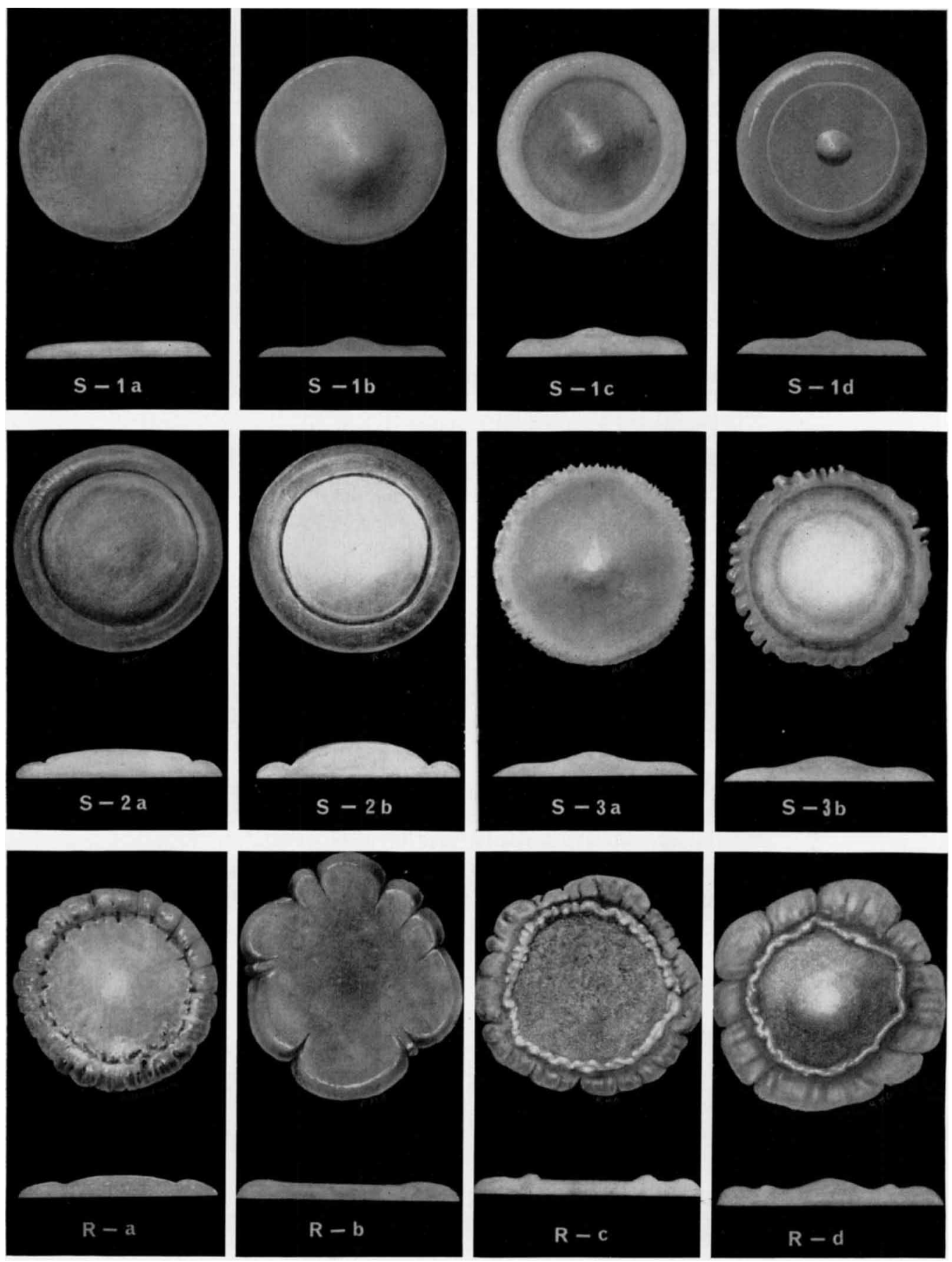
BRown, R. W. (1966). Color of cultures of Staphylococcus epidermidis determined by spectral reflectance colorimetry. J. Bact. $91,911$.

LosNegard, N. \& Oeding, P. (1963). Immunochemical studies on polysaccharides from Staphylococcus epidermidis. Acta path. microbiol. scand. 58, 493.

Manual of Microbiological Methods (1957). New York: McGraw-Hill Book Co., Inc.

MARKS, J. (1952). Recognition of pathogenic staphylococci: with notes on non-specific staphylococcal haemolysin. J. Path. Bact. 64, 175.

PAGE, L. A. (1962). Acetylmethylcarbinol production and the classification of aeromonads associated with ulcerative diseases of ectothermic vertebrates. J. Bact. 84, 772.

SANDVIK, O. (1962). Studies on Casein Precipitating Enzymes of Aerobic and Facultatively Anaerobic Bacteria, p. 116. The Veterinary College of Norway, Oslo.

SANDVIK, O. \& BROWN, R. W. (1965). Spectrophotometric characterization of pigments produced by Staphylococcus epidermidis strains isolated from bovine udders. J. Bact. 89, 1201.

SANDVIK, O. \& Fossum, K. (1965). The classification of certain members of the family Micrococcaceae by serologic differentiation of their proteolytic enzymes. Am. J. Vet. Res. 26, 357.

Shaw, C., StrTt, J. M. \& Cowan, S. T. (1951). Staphylococci and their classification. J. gen. Microbiol. 5, 1010.

\section{EXPLANATION OF PLATE}

Drawings of the colonial types produced by cultures of Staphylococcus epidermidis isolated from the milk of infected bovine udders. All colonial forms were drawn the same size, although isolated colonies of those illustrated were $1 \cdot 75-2.75 \mathrm{~mm}$. in diameter. S-2b colonies usually were smaller. Type s-1 colonies are not illustrated because they appeared similar to type $\mathrm{S}-1 \mathrm{a}$ and $\mathrm{c}$ except for being smaller $(1 \cdot 0-1.5 \mathrm{~mm}$. in diameter). 